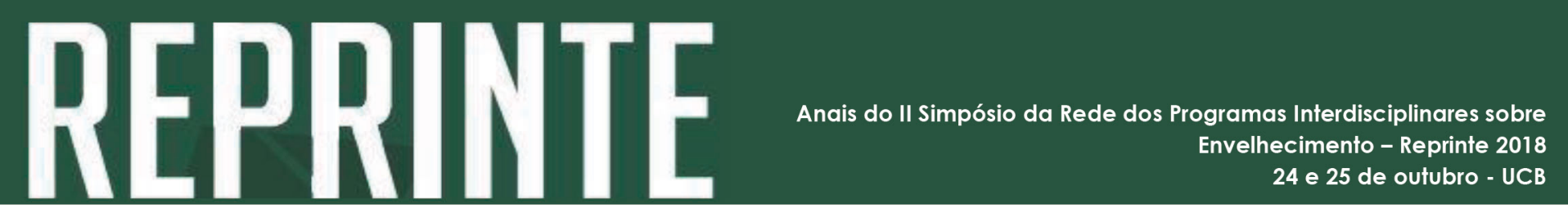

http://dx.doi.org/10.5335/rbceh.v16i1.9831

\title{
6) Relação entre sarcopenia e cognição: avaliação de seguimento do estudo FIBRA em Ermelino Matarazzo e Campinas
}

\author{
Gabriela Cabett Cipolli'; Ivan Aprahamian²; Deusivânvia Vieira da Silva Falcão3; \\ Flávia Silva Arbex Borim ${ }^{4}$; Anita Liberalesso Neri"; Meire Cachioni ${ }^{5}$; \\ Ruth Caldeira de Melo ${ }^{5}$; Samila Sathler Tavares Batistini ${ }^{5}$ Mônica Sanches Yassuda ${ }^{5}$
}

\section{Resumo}

A ligação entre a sarcopenia e a cognição ainda não foi completamente avaliada, especialmente entre os longevos.

Palavras-chave: Envelhecimento. Sarcopenia. Cognição.

\section{Introdução}

A sarcopenia é uma síndrome caracterizada pela perda progressiva e generalizada de massa e força muscular esquelética (MORLEY, 2012). A identificação da sarcopenia na população idosa é essencial no contexto da Gerontologia para que sejam planejadas intervenções, reduzindo a ocorrência de eventos adversos à saúde, como incapacidade física, redução na qualidade de vida e, morte (CRUZ-JENTOFT et al, 2010). Recentemente, a literatura tem demonstrado que pode existir uma relação entre a sarcopenia e o desempenho cognitivo (NISHIGUCHI, 2015).

1 Gerontóloga. Mestranda em Gerontologia, Escola de Artes, Ciências e Humanidades da Universidade de São Paulo. Endereço para correspondência: R. Arlindo Béttio, 1000, Ermelino Matarazzo São Paulo - SP, Brasil. CEP: 03828-000. São Paulo, Brasil. Email: gabriela.cipolli @usp.br

2 Médico especialista em Clínica Médica, Geriatria e Psiquiatria. Professor na Faculdade de Medicina de Jundiaí, São Paulo. E-mail: ivan.aprahamian@gmail.com

3 Psicóloga. Professora na. Escola de Artes, Ciências e Humanidade da Universidade de São Paulo, São Paulo. Email: deusivaniafalcao@gmail.com

4 Professoras do Programa de pós-graduação em Gerontologia UNICAMP. E-mails (na ordem em que aparecem): flarbex@hotmail.com; anitalbn@uol.com.br

5 Professoras na Escola de Artes, Ciências e Humanidade da Universidade de São Paulo, São Paulo. Emails (na ordem em que aparecem): meirec@usp.br; ruth.melo@usp.br; samilabatistoni@gmail.com; yassuda@usp.br 


\title{
Objetivo
}

Examinar se a relação entre sarcopenia e cognição é observada quando diferentes estratégias são utilizadas para o diagnóstico de sarcopenia.

\section{Método}

Estudo de corte transversal que utilizou os dados da segunda avaliação do Estudo da Fragilidade em Idosos Brasileiros (FIBRA) em Ermelino Matarazzo e Campinas. A amostra foi submetida a uma única sessão de coleta de dados em ambiente domiciliar, com protocolo que incluiu variáveis sociodemográficas, cognitivas (Mini-Exame do Estado Mental - MEEM e Mini Addenbrooke - Mini-ACE), medidas antropométricas, velocidade da marcha e força de preensão manual. Massa muscular foi calculada usando a fórmula de Lee (RECH et al, 2011). Compuseram a amostra idosos com preservação cognitiva no MEEM. Resultados: A amostra foi composta por 205 idosos de 80 anos ou mais, e 38 idosos $(19,1 \%)$ foram identificados com sarcopenia, segundo os critérios do European Working Group on Sarcopenia in Older People's (EWGSOP) [2]. Idosos com sarcopenia eram mais velhos e apresentaram pior desempenho no escore total do MEEM, da Mini-ACE e na recordação tardia deste último instrumento.

\section{Conclusões}

A sarcopenia parece apresentar repercussões negativas sobre a cognição de idosos longevos.

\section{Relationship between sarcopenia and cognition: follow-up evaluation of the FIBRA study in Ermelino Matarazzo and Campinas}

\begin{abstract}
The link between sarcopenia and cognition not yet been thoroughly evaluated, especially among the oldest old.
\end{abstract}

Keywords: Aging. Sarcopenia. Cognition.

\section{Referências}

CRUZ-JENTOFT, A. J. et al. Sarcopenia: european consensus on definition and diagnosis. Age and ageing, Cidade, v. 39, p. $412-423$, abr. 2010

MORLEY, John E. Sarcopenia in the elderly. Family practice, Cidade, v. 29, p. :i44-i48, jan. 2012.

NISHIGUCHI, S. et al. Differential Association of Frailty With Cognitive Decline and Sarcopenia in Community-Dwelling Older Adults. JAMDA, [S.L], v. 16, n. 2015, p. 120-124, ago. 2015.

RECH, C. R. et al. Validade de equações antropométricas para estimar a massa muscular em idosos. Rev Bras Cineantropom Desempenho Hum, v. 14, n. 1, p. 24-30, nov. 2011. 